\title{
SOME PROPERTIES OF BOREL SUBGROUPS OF REAL NUMBERS
}

\author{
BARTHELEMY LE GAC
}

\begin{abstract}
As a consequence of Souslin's theorem, we obtain the following: if $G$ and $H$ both are analvtic subgroups of $\mathbf{R}$ such that $G+H=\mathbf{R}$ and $G \cap H=\{0\}$. then either $G=\mathbf{R}$ or $G=\{0\}$. Next we obtain some measure and topological propertie: for uncountable proper Borel subgroups of reals. Finally, we prove that if $E$ is a vector subspace of $\mathbf{R}$ over the rationals which admits an uncountable Borel basis. then there exists no Polish topology on $E$ such that $E$ is a topological group with the given Borel structure generated by the open sets.
\end{abstract}

1. Introduction. In [12], J. von Neumann constructed a set $A$ of real numbers which is algebraically independent (over the field of rational numbers $\mathbf{Q}$ ) and has the power of continuum. The elements of $A$ are the reals $f(x)$ given by

$$
f(x)=\sum_{n=0}^{x} 2^{2^{\ln n n} /} / 2^{2^{n 2}} \cdot \quad x>0 .
$$

This function $f$ is strictly increasing. so one-one and Borel: hence $A$ is a Borel set by Lusin's theorem [8, p. 176]. If $P$ is a proper nonvoid perfect compact subset of $A$. the vector subspace $G$ of $\mathbf{R}$ over the rationals generated by $P$ gives us an example of an uncountable proper Borel subgroup (in fact a $\sigma$-compact one) of the reals. More generally, J. Mycielski proved in [11, Theorem 2] that every nonvoid perfect set of reals contains a nonvoid algebraically independent perfect subset; this is the key theoretic tool for the construction of such subgroups of $\mathbf{R}$. Finally, let us recall that any proper analytic subgroup of $\mathbf{R}$, both is a meager and a null set. (But note that a null subgroup of the reals is not always a meager set, see [4].)

\section{A consequence of Souslin's theorem.}

THEOREM 1. If $G$ and $H$ both are analytic subgroups of $\mathbf{R}$ such that $G+H=\mathbf{R}$ and $G \cap H=\{0\}$, then either $G=\mathbf{R}$ or $G=\{0\}$.

Proof. We equip both $G$ and $H$ with their relativized Borel structure. The map $f$ of $G \times H$ onto $\mathbf{R}$ defined by $f(x, y)=x+y$ is one-one and Borel; hence it is a Borel isomorphism, in virtue of Souslin's theorem [3, p. 135]. Let $g$ denote the inverse map of $f$; we have $g(t)=(x(t), y(t))$, for every real $t$. Hence both $x$ and $y$ are Borel

Received by the editors June 15, 1982

1980 Mathematics Suhject (lassification. Primary 28A05, 54H05: Secondary ()4A15, 22A49. $28(10$. $54 \mathrm{E} 50$

Ke'l nords and phrases. Borel subgroups of real numbers, analytic sets. Souslin's theorem. quasiinvariant measures, topological groups, locally compact and Polish topologies, vector subspaces of $\mathbf{R}$ over the rationals. 
group homomorphisms; so they are continuous, by virtue of a theorem by Banach which asserts that every Borel homomorphism of a Polish group $E$ to a separable metric group $F$ is continuous [1, Theorem 4, p. 23]. Then both $G$ and $H$ are closed in R. But one knows that a closed proper subgroup of the reals takes the form of an $a \mathbf{Z}$, for some real $a$. Now we conclude that either $G=\mathbf{R}$ or $G=\{0\}$.

REMARK. This theorem fails if $H$ is supposed only to be a null subgroup; see [5, p. 192], in which the authors exhibit such an $H$ which is moreover a Lusin set. if continuum hypothesis holds.

COROllaRY. Let $K$ be an analytic proper subfield of the reals. There is no analytic basis $A$ for $\mathbf{R}$ as a $K$ vector space.

Proof. Suppose that such an $A$ exists: hence for any $a$ in $A$, both the subspaces $G$ and $H$ generated respectively by $\{a\}$ and $A \backslash\{a\}$ are analytic subgroups of R. But this is impossible. by virtue of the preceding theorem.

In the case $K=\mathbf{Q}$, this corollary is in fact the classical result by Sierpinski: there is no Hamel basis in $\mathbf{R}$ which is an analytic set [13. p. 110].

Let us remark that if $K$ is a nonanalytic subfield of the reals, there may exist analytic bases for $\mathbf{R}$ as a $K$ vector space: see [3]. for an example with such a countable (infinite) basis.

3. A measure theoretic result. Throughout this section. we denote by $G$ an uncountable proper Borel subgroup of the reals, equipped with its given Borel structure (the relativized one).

THEOREM 2. There exists no nonzero Borel measure $\mu$ on $G$ which is quasi-invariant ( for the action of $G$ on $G$ ).

Proof. Let us suppose that such a measure $\mu$ exists on $G$. By the Weil-Mackey theorem [9. p. 146], there exists a unique locally compact second countable topology on $G$ such that $G$ is a topological group with the given Borel structure generated by the open sets. This topology is not discrete. since $G$ is uncountable. As a locally compact abelian group, $G$ is (topologically) isomorphic to a direct product $\mathbf{R}^{\prime \prime} \times H$. in which $H$ contains a compact open subgroup [6. Theorem 3.2. p. 69]. We have necessarily $n=0$, otherwise we can inject the connected group $\mathbf{R}^{n} \times\{0\}$ into $G$. Hence $G$ is isomorphic to $H$, and so it contains a compact open subgroup $K$; but this $K$ will also be a compact subgroup of $\mathbf{R}$. which implies $K=\{0\}$. Now the zero subgroup is open in $G$, which is impossible since the topology on $G$ is not the discrete one.

COROllary. There exists no locally compact topology on $G$ such that $G$ is a topological group with the given Borel structure generated by the open sets.

Proof. If such a topology exists on $G$, it contains a compact open subgroup $K$, by the same argument as above. This $K$ cannot be uncountable, in virtue of the precedent theorem; hence $K$ is finite, since it is both compact and countable; so $K$ is the zero subgroup in the reals. Now the group $G$ both is uncountable and discrete, which is impossible since the Borel sets in $G$ are not the whole subsets of $G$. 
4. In this last section, we are concerned only with vector subspaces of $\mathbf{R}$ over the rationals which admit Borel bases; such vector subspaces are Borel sets in $\mathbf{R}$; this is a result by $R$. D. Mauldin [10, p. 263]; using the same argument, we also obtain the following.

Proposition. Let $E$ and $E^{\prime}$ both be vector subspaces of $\mathbf{R}$, over the rationals, both of which admit respective Borel bases $B$ and $B^{\prime}$ such that $\operatorname{card}(B)=\operatorname{card}\left(B^{\prime}\right)$. Then $E$ and $E^{\prime}$ are isomorphic, as Borel (standard) groups.

Proof. We may restrict our attention to the case in which both $B$ and $B^{\prime}$ have the power of continuum. For every integer $n>0$, let $B_{n}$ be the Borel subset of the $n$-copies product $B^{n}$, the elements of which are the $n$-tuples of all different basis vectors. Let us define a map $f_{n}$ of $B_{n} \times \mathbf{Q}^{n}$ to $\mathbf{R}$ by

$$
f_{n}\left(b_{1}, \ldots, b_{n} ; r_{1}, \ldots, r_{n}\right)=\sum r_{i} b_{i} .
$$

Hence the set $E_{n}=f_{n}\left(B_{n} \times \mathbf{Q}^{n}\right)$ is Borel (Mauldin, [10]), and $E$ is equal to the increasing union of the sequence of Borel sets $E_{n}$. Using a result by $\mathrm{R}$. R. Kallman [7, Proposition 7.2, p. 240], we can obtain a Borel map $s_{n}$ of $E_{n}$ to $B_{n} \times \mathbf{Q}^{n}$ such that if

$$
s_{n}(t)=\left(b_{1}(t), \ldots, b_{n}(t) ; r_{1}(t), \ldots, r_{n}(t)\right) .
$$

then we have $\Sigma r_{i}(t) b_{i}(t)=t$, for all $t$ in $E_{n}$.

Let us consider now both the bases $B$ and $B^{\prime}$; there exists a Borel isomorphism $g$ of $B$ onto $B^{\prime}\left[8\right.$, p. 114]; we may naturally associate to it a $g_{n}$ of $B_{n} \times \mathbf{Q}^{n}$ onto $B_{n^{\prime}} \times \mathbf{Q}^{n}$, given by

$$
g_{n}\left(b_{1}, \ldots, b_{n} ; r_{1}, \ldots, r_{n}\right)=\left(g\left(b_{1}\right), \ldots, g\left(b_{n}\right) ; r_{1}, \ldots, r_{n}\right) .
$$

(We define $B_{n}^{\prime}$, $f_{n}^{\prime}$ and $E_{n}^{\prime}$ related to $E^{\prime}$ in the same way as the corresponding ones related to $E$.) Now we see that the composed map $h_{n}=f_{n}^{\prime} \circ g_{n} \circ s_{n}$ is one-one and Borel of $E_{n}$ onto $E_{n}^{\prime}$, and so it is a Borel isomorphism, by Souslin's thereom. Let $t$ in $E_{n}$ be given by $t=\Sigma r_{i} b_{i}$; thus $h_{n}(t)=\Sigma r_{i} g\left(b_{i}\right)$; so if we define a map $h$ of $E$ to $E^{\prime}$ by $h(t)=h_{n}(t)$, whenever $t$ is in $E_{n}$, we will obtain the desired Borel groups isomorphism.

THEOREM 3. Let $E$ be a vector subspace of $\mathbf{R}$ over the rationals which admits an uncountable Borel basis B. Then there exists no Polish topology on E such that $G$ is $a$ topological group with the given Borel structure generated by the open sets.

Proof. (The following proof is Mauldin's, the original one by the author being much more complicated.) Let us suppose that such a topology exists on $E$. Write $B$ as a strictly increasing union of a sequence of Borel sets $B_{n}$, and let $E_{n}$ be the corresponding vector subspace generated by $B_{n}$. We may assume that $E_{1}$ is dense in $E$ (equipped with its Polish topology). Each $E_{n}$ is an analytic subgroup of $E$ (in fact a Borel one), and therefore has the Baire property. One of the $E_{n}$ 's must be of the second category; hence this $E_{n}$ is closed and open in $E$, by [1, Theorem 1, p. 21]. It follows that this $E_{n}$ is all of $E$, since it is also dense in $E$. 
Question. Is there an uncountable proper Borel subgroup $G$ of $\mathbf{R}$ and a Polish topology on $G$ such that $G$ is a topological group with the given Borel structure generated by the open sets?

(If such a topology exists on a certain $G$, it is necessarily unique, by [1. Theorem 4 , p. 23].)

ACKnowledgements. The author would like to thank Professors Jan Mycielski. Robert R. Kallman and R. Daniel Mauldin for their helpful comments and suggestions: so this paper has improved considerably.

\section{REFERENCES}

1. S. Banach. Theoric des operations linéarres, Warsaw. 1932.

2. A. Bialynicki-Birula. (On subficlds of countahle codimension. Proc. Amer. Math. Soc. 35 (1972), $354-356$

3. N. Bourbaki, Topologete génerale. 2nd ed.. ('h. IX. Hermann. Paris. 1958.

4. P. Erdös, Some remarhs on suhgroups of real numbers. Colloq. Math. 42 (1979). 119-120.

5. P. Erdös. K. Kunen and R. Daniel Mauldin. Some additite properties of real numbers. Fund. Math 113 (1981). 187-199.

6. A. (juichardet. Analise harmonique commutatice. Dunod. Paris. 1968.

7. Rohert R. Kallman, Certain quotient spaces are (ountahly separated. III. J. Funct. Anal. 22 (1976), $225-241$

X. N. Lusin. Les ensembles analitiques, 2nd ed.. Chelsea. New York. 1972

9. (j. W'. Mackey. Borel structure's in groups and their duals. Trans. Amer. Math. Soc. 85 (1957). $134-165$

10. R. Daniel Mauldin. (n the Borel suhspaces of algehraic structures, Indiana Univ. Math. J. 29 (198()), $261-265$

11. J. Mycielski, Algehraic independence and measure. Fund. Math. 61 (1967), 165-169.

12. J. von Neumann. Sistem algehratsch unahhängiger Zahlen. Math. Ann. 99 (1928), 134-141

13. W. Sierpinski. Sur la question de la mesurahilite de la hase de M. Hamel. Fund. Math. 1 (1920). $104-111$

U. E. R. de Mathematiques. Universite de Provence, 3, Pi.ace Victor-Hugo, 13331 Marshili.l. CeDex 3, France 